\title{
Overshadowing between two contextual stimuli in rats
}

\author{
TAKASHI TAKIGASAKI' \\ Department of Educational Psychology, School of Education. Waseda University, Shinjuku-ku, \\ Tokyo 169
}

\begin{abstract}
The purpose of this experiment was to investigate whether overshadowing occurred between two contextual stimuli (light and tone) in Pavlovian conditioning of rats. Thirty-two albino rats, randomly assigned to four groups, were individually placed in a chamber, in which light, tone or both served as contextual stimuli. A single electric shock (1 $\mathrm{mA}, 2-\mathrm{s})$ was delivered to the floor during once-a-day $5 \mathrm{~min}$ sessions for 5 consecutive days (conditioning phase). The day after finishing the conditioning phase, each was placed for $5 \mathrm{~min}$ in the chamber, and light or tone was presented as a contextual stimulus (test phase). The group of rats exposed to both stimuli during the conditioning sessions but to light only during the test session (LT/L) froze less frequently in the test phase than in the conditioning phase. The other three groups (LTT, T/T, and L/L) froze in the test phase as frequently as in the conditioning phase. These results suggest that the tone overshadowed a light when both of them were presented together as contextual stimuli of conditioning.
\end{abstract}

Key words: overshadowing, contextual stimulus, freezing response, electric shock, rats.

In a Pavlovian conditioning, an animal learns not only the association between CS and US, but also between background stimuli and US. For example when US is presented, the animal is consistently exposed to experimental background such as experimental chamber, masking noise, and room light. Although these contextual stimuli are constantly present throughout the experiment and are unpredictive of the US, these stimuli may be associated with the US and control behavior of animals.

Previous studies suggested that the contextual stimuli may act like CSs. Association between the contextual stimulus and the US was well documented as an effect referred to as "the US preexposure effect" (Baker \& Mackintosh,1979; Balsam \& Schwartz,1981; Randich \& LoLordo,1979). Analyses of video-taped records of animal behavior revealed that the contextual stimuli were associated with the US (Rescorla, Durlach, \& Grau,1985; Fanselow, 1990). It would be

\footnotetext{
' I would like to thank Takayuki Koizumi and Yasushi Miyabe for their assistance in the data collection.
}

then expected that contextual stimuli may similarly cause phenomena such as overshadowing, blocking, and latent inhibition which have been well known as caused by CSs.

When two CSs are presented together with US, one of the CSs usually elicits greater $C R$ than the other; association between the more salient CS and US interferes with, or overshadows, the association between the less salient CS and the US. This phenomenon, referred to as overshadowing, is one of the primary characteristics of conditioning with two or more CSs presented in a compound fashion.

Similarly, overshadowing occurs between two contextual stimuli presented in a compound fashion. Baker, Singh, and Bindra (1985) reported that one contextual stimulus (tone) overshadowed the other contextual stimulus (experimental chamber) in rats. They used baseline suppression ratio of lever press for food, as a measure of contextual conditioning. However, their measure does not directly reflect the associations between the contextual stimuli and US.

The present experiment investigated whether two contextual stimuli (tone and 
light) presented in a compound fashion cause the overshadowing phenomenon. In the present study, frequency of rats' freezing response, directly controlled by contextual stimuli associated with US (electric shock), was used as a measure of contextual conditioning,

\section{Method}

Subjects. Subjects were 32 naive male Wistar albino rats, purchased from Nippon Bio-Supp. Center (Bunkyo-ku, Tokyo). They were housed individually and had free access to food and water in their home cages. Prior to the experiment, they received handling procedure $(10 \mathrm{~min}$ a day) and were familiarized with transportation from their home cages to the laboratory for 5 days. At the start of the experiment, they were 9 to 10 weeks old and weighed between 250 and 380 $\mathrm{g}$ (mean weight $=316 \mathrm{~g})$. Daily sessions were conducted during the lighted portion of a 12:12-h light : dark cycle (light on at 8.00 a.m.: about $50 \mathrm{~lx}$ in their home cages; light off at 8.00 p.m.: completely dark).

Apparatus. The size of the test chamber was $27 \times 18 \times 20 \mathrm{~cm}$. The ceiling and the front and back walls were made out of clear acrylic plastics, and the side walls were made by stainless steels. The floor was formed by a grid of 17 stainless steel rods $(5 \mathrm{~mm}$ in diameter and positioned $1.5 \mathrm{~cm}$ center to center) connected to a shock generator ( $O$ 'Hara \& Co. Ltd.) which provided an alternating scrambled shock (1-mA, 2-s). A 1.8-kHz, $65-\mathrm{dB}$ tone could be presented in the chamber through a speaker mounted outside of the side wall. A $15 \mathrm{~W}$ fluorescent lamp mounted $15 \mathrm{~cm}$ above the top of the back wall continuously and indirectly illuminated the test chamber. A $20 \mathrm{~W}$ fluorescent lamp mounted $15 \mathrm{~cm}$ above the top of the front wall directly illuminated the chamber. The chamber was cleaned with wet towels between sessions.
An $8 \mathrm{~mm}$ video system (Sony CCDV800) monitored and recorded rats' behavior. The test chamber and the video camera were placed in a sound-attenuating chest with a video monitor located outside the chest.

Procedure. The subjects were randomly divided into four groups prior to the experiment. On Day 1-5 (conditioning phase), all rats were individually placed in the test chamber. They received a single 1-mA, 2-s shock, 180-s after they were placed in the chamber and then returned to the home cages in a session of $5 \mathrm{~min}$. For the rats in LightTone/Light (LT/L) and Light-Tone/Tone (LT/T) groups, both contextual stimuli, the light and the tone, were presented throughout the sessions. The light stimulus was provided by the $20 \mathrm{~W}$ and $15 \mathrm{~W}$ fluorescent lamps (approximately $350 \mathrm{~lx}$ at the chamber floor) and the tone stimulus was presented by the speaker $(1.8-\mathrm{kHz}, 65-\mathrm{dB})$. The contextual stimulus of the light was used for the rats in Light/Light (L/L) group, and that of the tone was used for the rats in Tone/Tone (T/T) group. The contextual stimuli turned on when each rat was placed in the chamber and turned off after it was returned to the home cage.

On Day 6 (test phase), the rats were placed in the chamber for $5 \mathrm{~min}$ without shock. The rats in Groups LT/L and L/L were tested with the light alone, and those in Groups LT/T and T/T were tested with the tone alone.

An observer who was blind to the experimental conditions scored freezing behavior of the rats in every 5-s. The rats' behavior were recorded by a video system during the sessions, and the samples for observation were from the first $180 \mathrm{~s}$ ( 36 samples per rat) in each of 6 sessions. The observer judged if the rats froze. Freezing was defined as the absence of visible movement with hindlimbs contracted and forelimbs extended. Movements due to respiration were disre- 
garded (Fanselow, 1990). This is essentially the same as "crouching" defined by Blanchard, Dielman. and Blanchard (1968). A second observer, who was also blind to the experimental conditions, rescored the freezing response in the videotapes. Interobserver reliability was calculated by dividing the total number of scores which both observers scored either as freezing or not by the total number of samples. The agreement on scores between the observers was $92.3 \%$.

\section{Results}

Analyses were conducted using the data scored by the first observer. Figure 1 shows percentages of samples scored as freezing against 288 samples ( 36 samples $\times 8$ rats in each group) as a function of experimental days. In all four groups, the freezing response increased over 5 days of the conditioning phase. At the end of the conditioning phase (Day 5), the frequencies of freezing increased to fifty percent of the samples. A two-way analysis of variance, with Day (Day

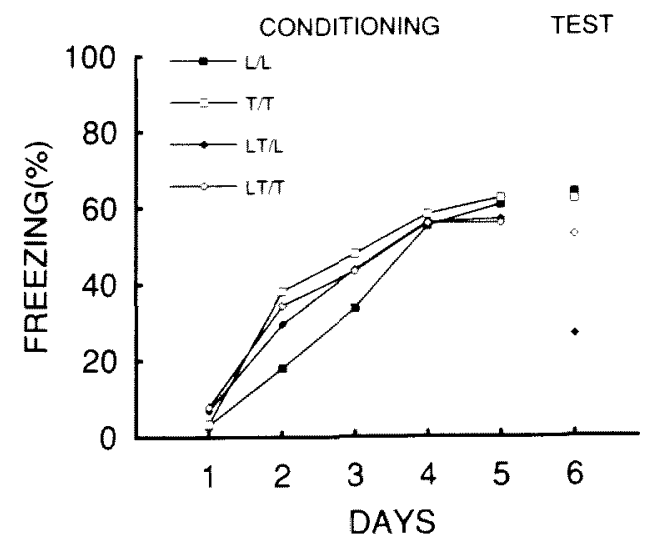

Figure 1. The percentage of freezing during the conditioning phase (Day 1 to 5) and the test phase (Day 6) in each group. The rats experienced the electric shock with the presentation of light $(\mathrm{L} / \mathrm{L})$, tone (T/T) or both (LT/L, LT/T) in the conditioning phase, and placed in the chamber with the presentation of light $(\mathrm{L} / \mathrm{L}, \mathrm{LT} / \mathrm{L})$ or tone $(\mathrm{T} / \mathrm{T}, \mathrm{LT} / \mathrm{T})$ in the test phase.
1 to Day 5) and group (L/L, T/T, LT/L, and $\mathrm{LT} / \mathrm{T})$ as variables, was conducted. A significant effect was found for Day $[F(4,140)=40.80, p<.001]$. The effects for group and the interaction were not significant $(p>1)$.

A one-way analysis of variance of freezing score on Day 6 (test phase) revealed that the group effect was significant $[F(3,28)=5.26, p<.01]$. Subsequent $t$-test (Bonferroni) indicated that the numbers of freezing in the rats in Group LT/L were significantly less than those in the rats in other groups $\left(p_{s}<.05\right)$. A paired $t$-tests revealed that there was no difference in freezing between Days 5 and 6 in Groups L/L, T/T, and LT/T $\left(p_{s}>1\right)$, whereas the numbers of freezing in the rats in Group LT/L were significantly less on Day 6 than on Day 5 [ $t$ (7) $=4.49, p<.01]$. Thus, when two contextual stimuli (the light and tone) were presented in a compound fashion, the tone was effectively associated with the shock and overshadowed the light.

Figure 2 shows percentages of samples

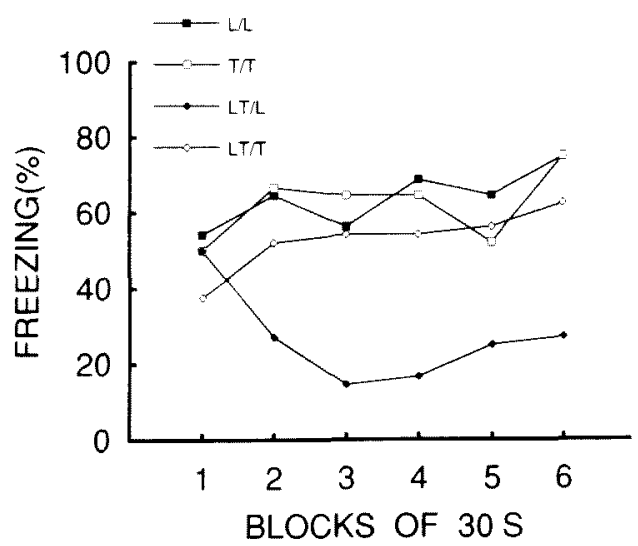

Figure 2. The percentage of freezing in blocks of 30 s during the test phase (Day 6) in each group. The rats experienced the electric shock with the presentation of light (L/L), tone (T/T) or both (LT/L, LT/T) for 5 days, and placed in the chamber with the presentation of light $(\mathrm{L} / \mathrm{L}, \mathrm{LT} / \mathrm{L})$ or tone $(\mathrm{T} / \mathrm{T}, \mathrm{LT} / \mathrm{T})$ in the test phase. 
scored as freezing on Day 6 against 48 samples ( 6 samples $\times 8$ rats in each group) as a function of blocks of $30 \mathrm{~s}$. A two-way analysis of variance, with block (Block 1 to Block 6) and group (L/L, T/T, LT/L, and LT/T) as variables, was conducted. A significant effect was found for groups $[F(3,168)=16.80$, $p<.001]$. The effects for blocks and the group $\times$ block interaction were not significant $(p>1)$. Although a significant effect was not found for blocks, the rats in Groups $L / L, T / T$, and LT/T froze most frequently immediately before a shock would have been delivered in the conditioning sessions. In contrast, the rats in Group LT/L froze most frequently in the first 30 seconds after being placed in the chamber, and gradually decreased freezing in the first half of the session.

\section{Discussion}

The overshadowing phenomenon occurred between two contextual stimuli (light and tone) in the conditioning with electric shock as US in rats. The light and the tone each presented alone as the contextual stimulus in Groups $\mathrm{L} / \mathrm{L}$ and $\mathrm{T} / \mathrm{T}$ were conditioned to the US. As shown in Figure 1, the rats in these groups froze more than fifty per cent of the samples in the test phase (Day 6). On the other hand, when these stimuli were presented in a compound fashion, the tone overshadowed the light. The rats in Group LT/L froze less frequently in the test phase than in sessions at the end of the conditioning phase. In other words, the light was not well associated with the electric shock in the conditioning phase in which it was compounded with the tone. In contrast, such an overshadowing effect was not observed in the rats in Group LT/T.

The asymmetrical overshadowing effect suggests that light is more salient than tone for rats when they are presented in a compound fashion. There may be at least two possible accounts for the difference in salience between these contextual stimuli. One account is that because the subjects had never experienced loud tones in their home cages, the $1.8-\mathrm{kHz}, 65-\mathrm{dB}$ tone used as the contextual stimulus in the present study might be strong enough to induce the subjects to predominantly attend to the tone. The other account is that because the subjects experienced the light (about $50 \mathrm{~lx}$ ) for 12 -h per day in their home cages, the association between the contextual light and the shock might not be well established in the experimental chamber. It has been shown that frequent preexposure to experimental context inhibits the context-shock conditioning in rats (Balaz, Capra, Kasprow, \& Miller, 1982; Fanselow, 1989). In the present study, it is quite conceivable that the light was inhibited to be associated with the shock due to the preexperimental experiences.

It is suggested that the contextual stimuli in the present experiment control rats' behavior more effectively than those presented with CSs. The finding that the overshadowing phenomenon occurred between two contextual stimuli supports the notion that the contextual stimuli may exert control over behavior in a similar manner as CSs (e.g., Blanchard \& Blanchard, 1969; Bolles \& Collier,1976; Bouton \& King,1983). However, no CS was presented in this experiment. Therefore, the contextual stimuli used in this experiment might be associated with the US more strongly than those presented as the background of CSs.

It should be noted that it was not only the contextual stimuli but also the placementshock interval and manipulations before experiments that might have controlled the behavior of rats in the present experiment. The analyses of behavior during Day 6 revealed that the rats in Groups $\mathrm{L} / \mathrm{L}, \mathrm{T} / \mathrm{T}$, and LT/T tended to freeze most frequently immediately before the shock. This suggests that 
the rats remembered the interval between the placement in the chamber and the shock. On the other hand, the rats in Group LT/L froze most frequently during the first few minutes after being placed in the chamber. This suggests that the rats associated the shock with the transportation from home cages to the laboratory. The effects of placement-shock interval and manipulations before experiments on animal behavior in laboratory should be further explored.

\section{References}

Baker, A. G. \& Mackintosh, N. J. 1979 Preexposure to the CS alone, US alone, or CS and US uncorrelated: Latent inhibition, blocking by context or learned imelevance? Learning and Motivation. 10, 278-294.

Baker, A. G., Singh, M. \& Bindra, D. 1985 Some effects of contextual conditioning and US predictability on Pavlovian conditioning. In P. D. Balsam \& A. Tomie (Eds.), Context and learning. Hillsdale, N.J.: Lawrence Erlbaum Associates. Pp. 73-103.

Balaz, M. A.. Capra, S., Kasprow, W. J., \& Miller, R. R. 1982 Latent inhibition of the conditioning context: Further evidence of contextual potentiation of retrieval in the absence of appreciable context-US associations. Animal Learning \& Behavior, 10, 242-248.

Balsam. P. D., \& Schwartz, A. L. 1981 Rapid contextual conditioning in autoshaping. Journal of Experimental Psychology: Animal Behavior Processes, 7, 382-393.
Blanchard, R. J., \& Blanchard, D. C. 1969 Crouching as an index of fear. Journal of Comparative and Physiological Psychology, 67, 370-375.

Blanchard, R. J, Dielman, T. E., \& Blanchard, D. C. 1968 Prolonged aftereffects of a single foot shock. Psychonomic Science, 10, 327-328.

Bolles, R. C., \& Collier, A. C. 1976 The effect of predictive cues on freezing in rats. Animal Learning \& Behavior, 4, 6-8.

Bouton, M. E., \& King, D. A. 1983 Contextual control of extinction of conditioned fear: Tests for the associative value of the context. Journal of Experimental Psychology: Animal Behavior Processes, 9, 248-265.

Fanselow, M. S. 1989 The adaptive function of conditioned defensive behavior: An ecological approach to Pavlovian stimulus substitution theory. In R. J. Blanchard, P. F. Brain, D. C. Blanchard, \& S. Parmigiani (Eds.), Ethoexperimental approaches to the study of behavior. Dordrecht, The Netherlands: Kluwer. Pp. $151-166$

Fanselow, M. S. 1990 Factors governing one-trial contextual conditioning. Animal Learning \& Behavior, 18, 264-270.

Randich. A., \& LoLordo, V. M. 1979 Associative and nonassociative theories of the UCS preexposure phenomenon: Implications for Pavlovian conditioning. Psychological Bulletin, 86, 523548.

Rescorla, R. A., Durlach, P. J., \& Grau, J. W. 1985 Contextual learning in Pavlovian conditioning. In P.D. Balsam \& A. Tomie (Eds.), Context and learning. Hillsdale, N. J.: Lawrence Erlbaum Associates. Pp. 23-56

(Received Sept. 24, 1993; accepted Sept. 3, 1994) 